\title{
Souris à hémoglobine humaine
}

L'intérêt qu'il y aurait à posséder des modèles murins des hémoglobinopathies a déjà été souligné lorsque nous avons rapporté les résultats de F. Costantini qui, en 1986, était parvenu à créer des souris transgéniques exprimant le gène $\beta$ humain $(\mathrm{m} / \mathrm{s}$ $n^{\circ} 1$, vol. 3, p. 49). Les animaux transgéniques souffraient d'une thalassémie $\beta$ et ne synthétisaient donc pas de chaînes de globine $\beta$ majeures murines; ils produisaient de la globine hybride de formule $\alpha_{2}$ souris $\beta_{2}^{\text {humaine. Des souris possédant le gène }}$ $\beta^{\text {s }}$ (c'est-à-dire codant pour la chaîne $\beta$ dont la mutation entraîne la falciformation spécifique de la drépanocytose) ont ensuite été créées. Malheureusement, elles ne reproduisaient pas le modèle de la maladie car aucune falciformation des hématies n'était observée à basse concentration en oxygène : la sous-unité $\alpha$ murine inhibait la précipitation de l'hémoglobine hybride $\alpha_{2}$ murine $\beta_{2}^{\text {s }}$ et la concentration en cette molécule était de toute façon trop faible pour lui permettre de précipiter. Plusieurs travaux récents, dont $\mathrm{m} / \mathrm{s}$ a également rendu compte $\left(\mathrm{m} / \mathrm{s} n^{\circ} 4\right.$, vol. 4 , p. 452), ont ultérieurement démontré que le gène de globine $\beta$ nécessitait, pour être normalement exprimé, la présence d'un élément d'ADN qui est situé à 45 kilopaires de bases ( $\mathrm{kpb}$ ) en amont de son promoteur. Cet élément a été « disséqué » par plusieurs équipes qui ont démontré qu'il suffisait d'ajouter environ de 0,8 à $3 \mathrm{kpb}$ à une construction génétique comportant un promoteur ubiquitaire pour conférer à ce dernier une spécificité érythroïde d'expression. Des chercheurs américains des laboratoires de R.L. Brinster et T. Asakura (Philadelphie, PA), T.M. Townes (Birmingham, AL) et R.D. Palmiter (Seattle, WA) viennent, sur ce principe, de créer des souris transgéniques pour, à la fois, les gènes $\alpha$ et $\beta$ de la globine humaine, tous deux stimulés par l'activateur amont du obtenues ont une concentration normale en hémoglobine, mais expriment autant de globine humaine que de globine murine, sous la forme de tétramères homologues $\left(\alpha_{2} \beta_{2}\right.$ murins et humains) et, en moindre quantité, hétérologue (c'est-à-dire contenant des chaînes des deux espèces). Le fait que ces animaux aient une concentration en hémoglobine normale indique qu'il existe un rétrocontrôle limitant l'accumulation de cette protéine, qu'il agisse sur la transcription des gènes, la traduction des messagers ou, peut-être plus probablement, la stabilité de la protéine dont la concentration est déjà saturante dans les conditions physiologiques habituelles. La prochaine étape consistera à créer des animaux synthétisant les tétramères $\alpha^{\text {humain }} \beta_{2}^{\mathrm{s}} \ldots$ et à vérifier que ces molécules sont capables de précipiter dans les hématies murines incubées à basse concentration en oxygène. Il faut remarquer que si la méthode utilisée reste celle de la publication récente [1], les animaux transgéniques obtenus ressembleront aux hétérozygotes pour la mutation drépanocytaire... et seront donc, de toute façon, relativement à l'abri des crises sévères de falciformation. Peut-être l'utilisation de souris thalassémiques permettrait-elle d'aboutir à des animaux dont la majorité de l'hémoglobine comporterait de la globine humaine drépanocytaire? Un tel résultat constituerait un avantage certain pour le test in vivo des médicaments destinés à inhiber la précipitation de l'hémoglobine mutée, la falciformation des hématies et ses conséquences pathologiques (c'est-à-dire les microthromboses).

A.K.

1. Behringer RR, Ryan TM, Reilly MP, et al. Synthesis of funtional human hemoglobin in transgenic mice. Science 1989; $245: 971-3$.
On peut être normal avec 44 chromosomes. Les translocations robertsoniennes sont dues à une fusion de deux chromosomes par leurs extrémités. Elles diminuent donc le nombre apparent des chromosomes sans perte de matériel. Équilibrées, elles ne créent en principe pas d'anomalies cliniques. Elles ne sont dangereuses que par le déséquilibre qu'elles peuvent provoquer à la génération suivante. A l'état hétérozygote, on évalue leur fréquence à environ 1 pour 1000 personnes. C'est dire que les homozygotes sont exceptionnels et qu'on peut s'attendre à en trouver surtout dans des unions consanguines. Dallapiccola et al. en Italie [1] ont eu l'occasion, chez un couple de cousins germains, de découvrir lors d'un examen prénatal au premier trimestre, un foetus porteur de 44 chromosomes par suite d'une fusion homozygote des chromosomes 14 et 21 . Après les hésitations qu'on imagine, il fut décidé de poursuivre la grossesse sous contrôle échographique. Un garçon est né dont le développement postnatal est apparemment normal. D'après les auteurs, on connaît cinq autres cas de translocations robertsoniennes homozygotes : un présentait, comme le cas décrit ci-dessus, une translocation $14 ; 21$, dont curieusement seul le père et non la mère était porteur ; les quatre autres, dont trois d'une même famille, avaient une translocation $13 ; 14$. Tous étaient phénotypiquement normaux. Si donc ces sujets à 44 chromosomes sont normaux du point de vue somatique, reste une interrogation sur leur fertilité, tout au moins pour les hommes, car les deux femmes porteuses de translocation $13 ; 14$ et en âge de procréer sont fertiles. Bien entendu, s'il existe une descendance, tous les enfants devraient être hétérozygotes pour la translocation.

[1. Dallapiccola B. et al. Prenatal Diagn 1989; 9 : 555-8.] 